\title{
En el umbral de la revolución en educación
}

La formación de maestros

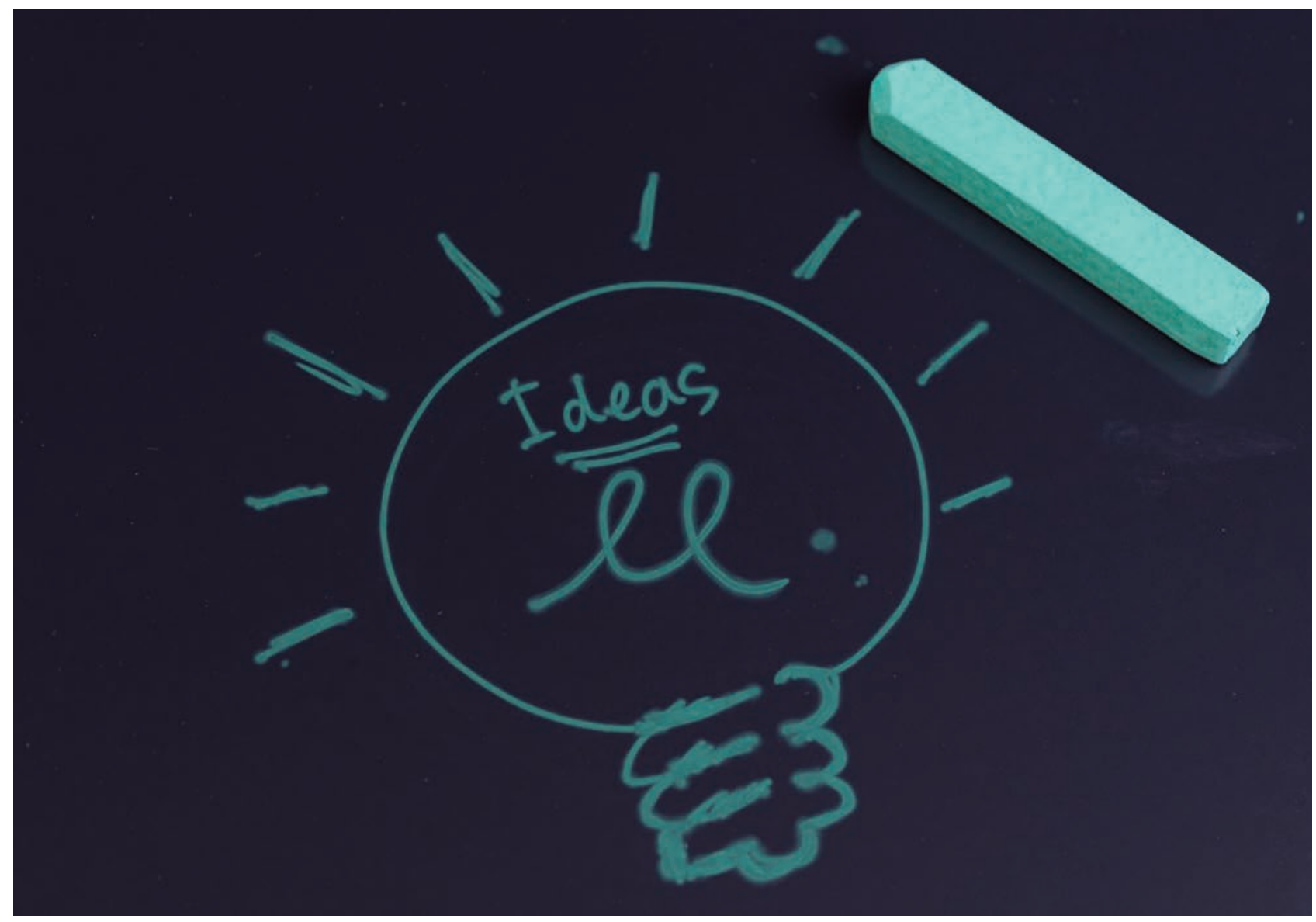

Este artículo presenta la situación del cambio en la educación derivado de la revolución tecnológica de este siglo. Analiza las competencias que los futuros alumnos tendrán que desarrollar para ejercer como ciudadanos activos en la sociedad del siglo XXI. Se detalla,

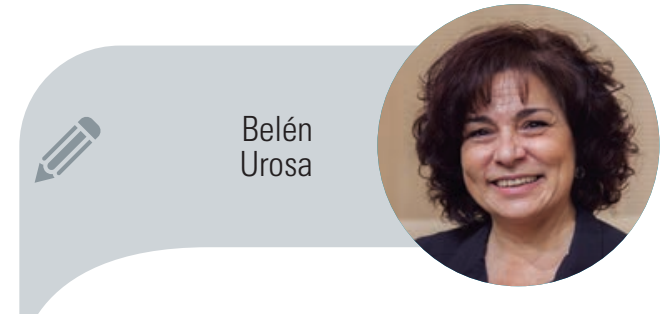

Universidad Pontificia Comillas burosa@comillas.edu además, la formación que como consecuencia deben tener los maestros que se necesitan para este nuevo contexto educativo y cómo debería cambiar su formación inicial. 
Acabamos de comenzar una nueva década en este siglo XXI y después de cómo han sido estos primeros 20 años da vértigo asomarnos a cómo serán los próximos ochenta. Siempre es difícil hacer prospectiva de lo que va a venir, parece que estuviéramos ante la bola de cristal que nos ayuda a imaginar y adivinar mágicamente los acontecimientos futuros. Pero, si miramos y analizamos lo que ha ido sucediendo en el transcurrir de este siglo puede que, la "adivinación" y el error en las predicciones se reduzcan de forma drástica.

Al igual que fue definitiva la revolución industrial en el siglo XX, la revolución tecnológica lo está siendo en el XXI. En el mundo vivimos actualmente 7.700 millones de personas, y de ellas tienen teléfono móvil ya más de 5.000 millones, usan internet más de 4.500 millones y son activas en redes sociales casi $3.500 \mathrm{mi}$ Ilones. Y la progresión en el uso de estas tecnologías es geométrica.

La tecnología ya ha cambiado el mundo, nuestras sociedades, la economía, la política, el mercado de trabajo, la salud..., como consecuencia de la manera en que nos comunicamos, nos informamos y claramente como consecuencia de la manera en la que aprendemos. Por tanto, no es necesario "adivinar", ya es una realidad, que aprendemos de forma diferente a como lo hacíamos antes y es claro que el avance en la tecnología hará que la próxima generación lo haga de forma también distinta a la actual. La sociedad y este nuevo mundo van a demandar habilidades y conocimientos distintos, para los que hasta ahora no hemos preparado a los alumnos, por lo que en consecuencia el cambio en el mundo educativo está asegurado.

En la era de la información, la comunicación y la conectividad, en el siglo del desarrollo de la inteligencia artificial, la automatización y los robots, no podemos dejar de pensar en los beneficios, pero también en los "peligros" que puede conllevar para las personas y su bienestar personal y social esta evolución. Existen nuevos perfiles profesionales y muchos de los perfiles existentes serán sustitui-

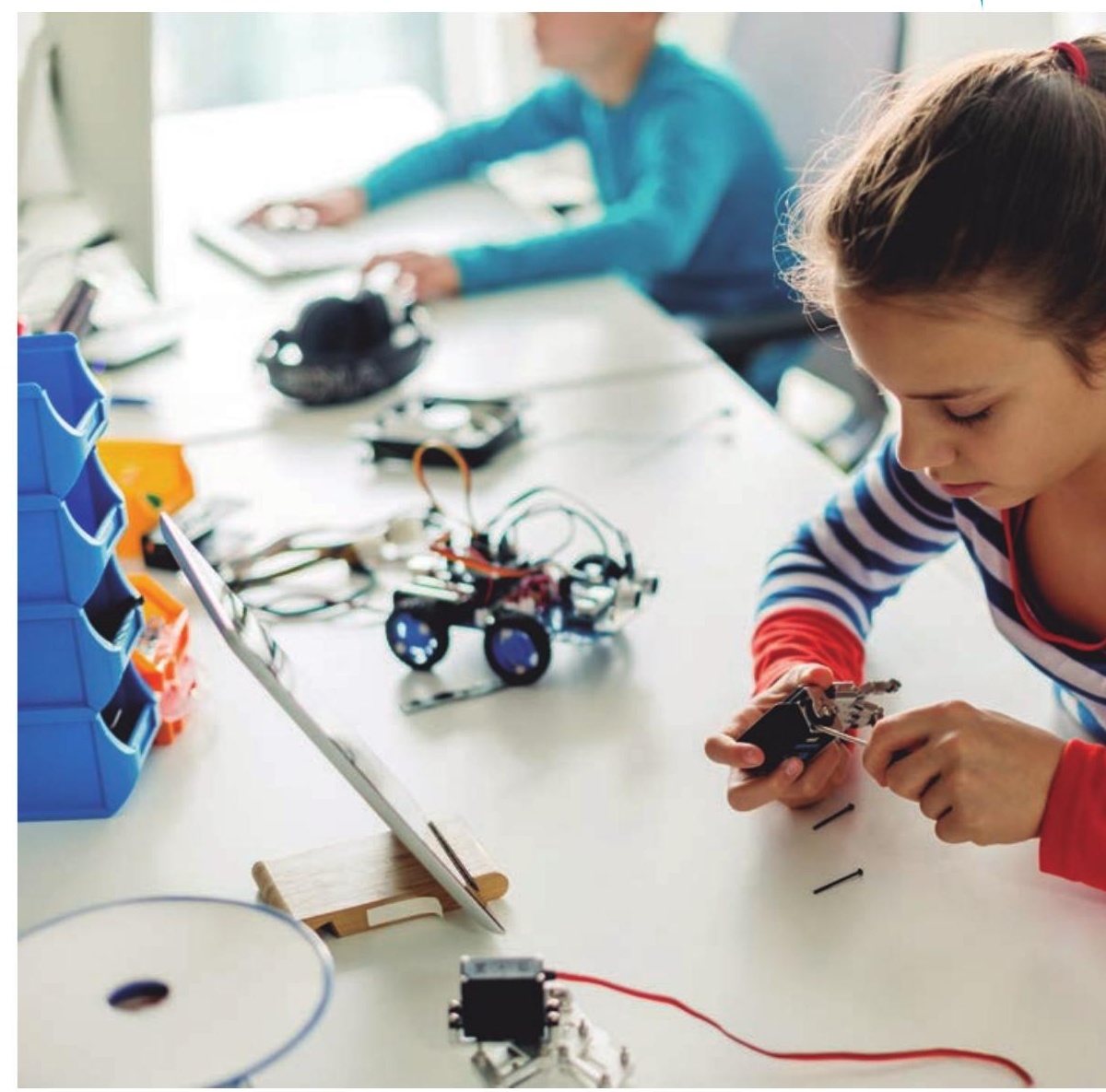

dos por máquinas. Las consecuencias que esto va a provocar serán parte de los grandes problemas a los que tendrán que enfrentarse las nuevas generaciones.

\section{Un cambio necesario}

No podemos formar a los alumnos en competencias que hacen mejor los robots, ellos memorizan y acumulan más información de la que somos capaces de retener las personas y realizan acciones automáticas también mejor, incluso pueden adaptar su respuesta en función de determinadas circunstancias. Las personas no podrán competir por puestos de trabajo donde se necesiten este tipo de competencias, ganarían siempre ellos. Tenemos que formar a los futuros ciudadanos para que utilicen las máquinas en la resolución de problemas complejos y su preparación debe centrarse en el desarrollo de competencias que hoy por hoy no parece que puedan realizar, al menos durante un tiempo, los robots. Si la cualificación profesional siempre ha sido importante, en un futuro próximo lo será aún más. El mayor peligro es que las personas que tengan una baja cualificación no puedan 


\author{
Nos encontramos en nuestras aulas con alumnos \\ que podríamos llamar “absentistas presenciales”, \\ que vienen al centro, están en la clase, pero \\ viven desconectados de lo que sucede en ella
}

llegar a ser ciudadanos activos porque los posibles trabajos que podrían desempeñar los lleguen a realizar tan solo máquinas. El riesgo de que se aumente aún más la brecha laboral ya existente, en función de la formación de las personas, es una realidad absoluta.

Pero no podemos pensar de forma negativa, de situaciones críticas nacen los retos y se producen las oportunidades y los mejores cambios. Las personas interactuamos con nuestro entorno, con otras personas, y esta interacción nos enriquece y nos mejora, aprendemos de esta relación y somos capaces de cooperar y colaborar produciendo aprendizajes colectivos y enriqueciendo nuestras respuestas y soluciones ante problemas complejos o situaciones inesperadas. Colectivamente podemos afrontar de mejor manera la incertidumbre. Hoy por hoy no hay máquinas que puedan aprender de y con otras máquinas.

Las personas podemos desarrollar nuestro pensamiento crítico y podemos evaluar y entender datos que son fruto de manipulaciones, podemos prepararnos para identificar "falsas noticias", incluso identificar la intencionalidad de influir en nosotros de los demás. También podemos imaginarnos situaciones nuevas, podemos utilizar nuestro pensamiento creativamente e inventar soluciones creativas y distintas a los problemas. Podemos valorar las consecuencias éticas de determinadas conductas y entender a los otros en base a la empatía. Las máquinas pueden simular sentimientos, pero no pueden sentir, no pueden ponerse en la mente y en el corazón del otro.
Debemos potenciar estas competencias que nos hacen humanos, nos diferencian y nos hacen mejores, y solo podemos hacerlo a través de la educación.

Sin embargo, el sistema educativo actual y el currículo de las diferentes etapas sigue considerando como elemento central los contenidos. Las leyes, decretos y órdenes, pueden tener en cuenta además los objetivos, competencias, metodologías y criterios de evaluación. Pero, la realidad de la mayoría de nuestros centros es que la gran cantidad de contenidos a trabajar en las distintas etapas hacen que sigamos centrándonos en estos más que en el desarrollo competencial. Que sigamos más preocupados por enseñar que por que nuestros alumnos aprendan más allá de la mera adquisición de estos contenidos. Este sistema educativo, fragmentado en asignaturas en el que prevalecen los conocimientos y se utiliza como recurso de aprendizaje principal el libro de texto, impide o al menos dificulta el que en nuestros centros se desarrollen actividades formativas con un enfoque más interdisciplinar, dirigidas a la resolución de problemas actuales y que impliquen la aplicación por parte de los alumnos de habilidades complejas y en consecuencia se puedan llevar a cabo aprendizajes más profundos y transversales.

Los profesores y los centros educativos nos encontramos con problemas que hace unos años no eran tan acuciantes. Hemos trabajado para que la educación fuera universal y que todos pudieran formarse, y esto ya es una realidad en los países desarrollados. Sin embargo, nos encontramos en nuestras aulas con alumnos que podríamos llamar "absentistas presenciales", que vienen al centro, están en la clase, pero viven desconectados de lo que sucede en ella. Según un estudio del Banco Mundial en la actualidad existen más de 100 millones de niños escolarizados en el mundo que no están aprendiendo. Lamentablemente cada vez son más los alumnos que muestran un total desinterés por su formación, les falta motivación, se aburren en clase y terminan siendo absentistas reales e incluso aban- 


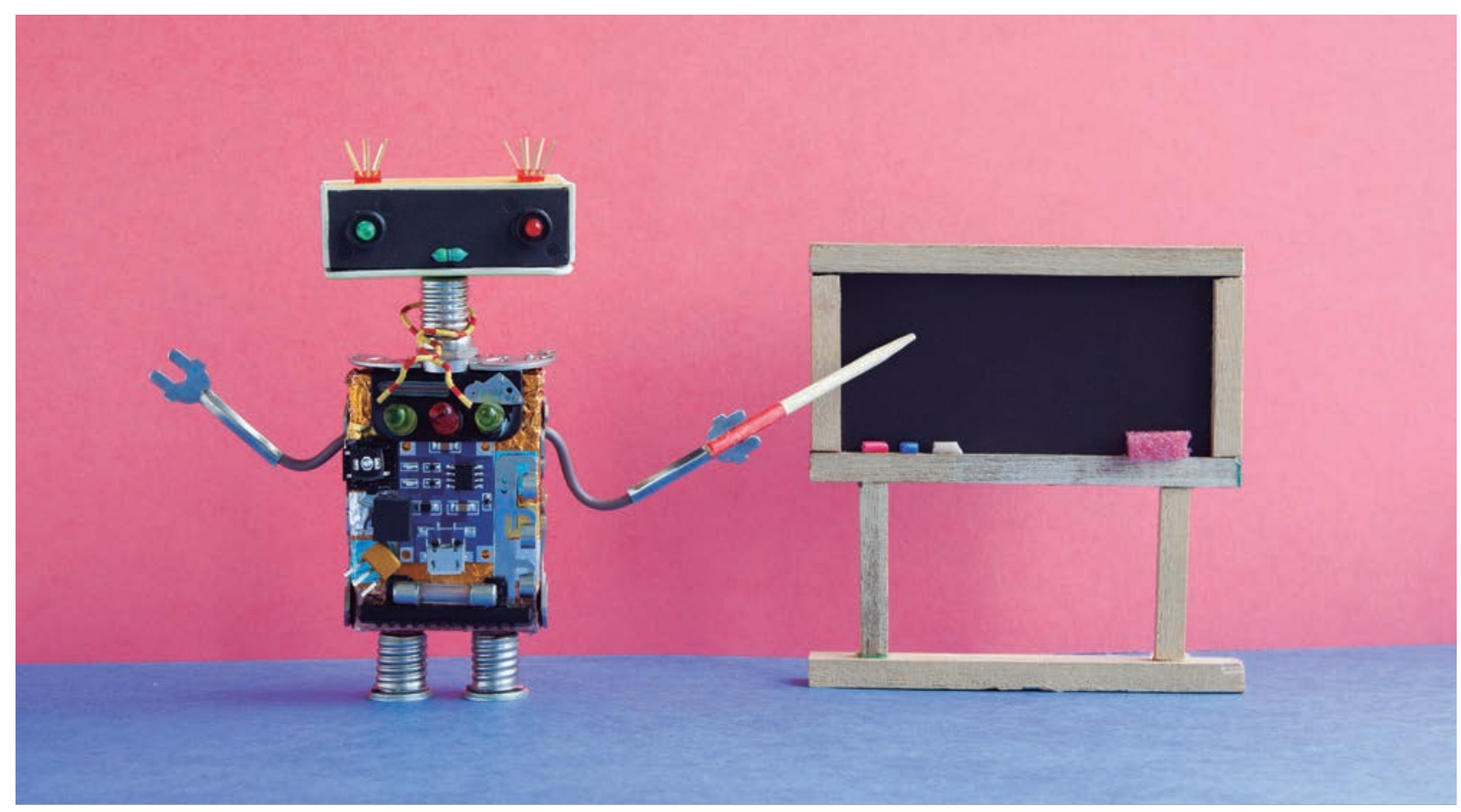

donando de forma temprana el sistema educativo. La orientación educativa hacia la transmisión del conocimiento no ayuda a que estos alumnos participen de forma activa en su formación. La percepción de que existe una baja conexión entre la realidad escolar y la vida futura de nuestros alumnos cada vez es más común en muchos de ellos.

Todo nos lleva a pensar en la necesidad urgente de un cambio en la educación. Un cambio profundo. Esto no significa cambiar una vez más las leyes, sino reflexionar sobre la realidad de nuestro sistema educativo. Pensar en cómo queremos que sean los ciudadanos del futuro, en qué conocimientos realmente son necesarios que adquieran porque serán la base de otros conocimientos y permitirán desarrollar procesos de pensamiento, y cuáles no les resultarán necesarios porque los tendrán al alcance de un clic o integrados en un chip que llevarán incorporado. Necesitamos reflexionar sobre cómo hacer que desarrollen las competencias que van a necesitar para el futuro incierto que les espera y dejar espacio en el currículo para su desarrollo. Necesitamos buscar cómo no perder a los alumnos por el camino y engancharlos a su formación e ilusionarlos con su futuro, pensando en su motivación pero también en las nuevas maneras de aprender. Y todo esto supone reformar los objetivos, el contenido, pero también las metodologías que empleamos. Necesitamos revolucionar de forma urgente la educación.

\section{Las competencias del siglo XXI}

En 2015, las Naciones Unidas establecieron un conjunto de 17 objetivos para el desarrollo sostenible (ODS) en el que están comprometidos más de 190 países. El objetivo 4 se refiere a la educación y debería marcar nuestro horizonte: "Garantizar una educación de calidad, inclusiva y equitativa, y promover las oportunidades de aprendizaje permanente para todos".

Teniendo en cuenta este horizonte, la educación debería centrarse fundamentalmente en que los estudiantes puedan desarrollar las competencias necesarias para el desempeño de una vida futura como ciudadanos activos. La educación siempre ha tenido como objetivo preparar para la vida, y esa vida va a suponer para los alumnos el que tengan que trabajar con un alto grado de incertidumbre, en situaciones en las que deberán colaborar con otros para hacer frente a los retos y problemas complejos de la vida real que corresponden a múltiples ámbitos (social, económico, político, ambiental...). Los retos e incertidumbres a los que deberán hacer frente las nuevas generaciones son los problemas derivados de la globalización, el cambio climático, la automatización, la crisis social, los problemas sociopolíticos, 


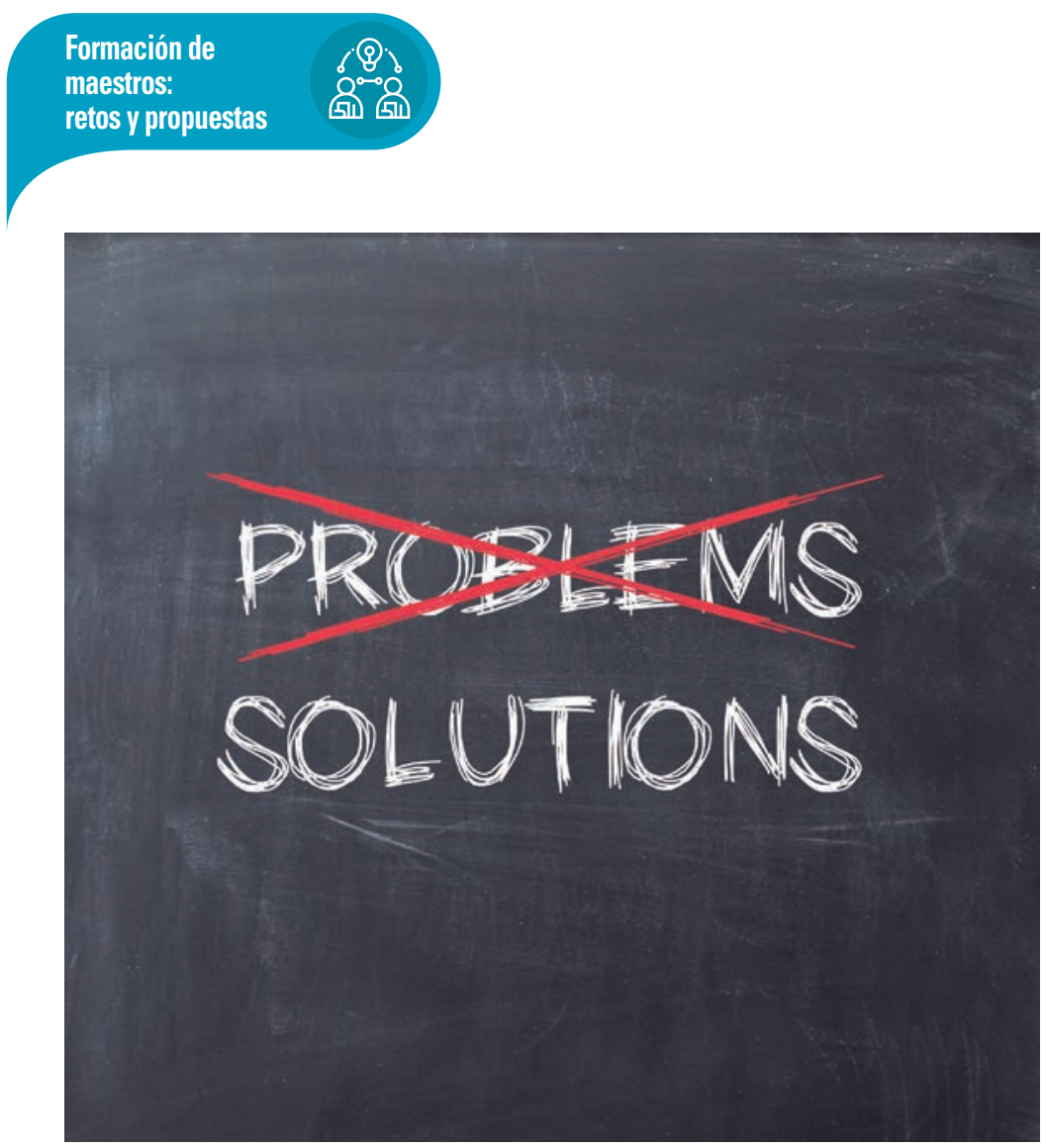

el envejecimiento, las desigualdades, las migraciones, los conflictos transnacionales, la pobreza, las enfermedades, la crisis energética..., por nombrar algunos. Deberán diseñar soluciones utilizando su pensamiento crítico y sus habilidades de búsqueda, selección y análisis de información, sopesando los pros y contras de distintas soluciones e imaginando con creatividad nuevos entornos o realidades.

Mucho se ha escrito desde el ámbito educativo sobre las habilidades futuras que deben tener los alumnos, incluso existen alianzas de distintas instituciones y ámbitos geográficos para el desarrollo de lo que vienen denominándose "competencias del siglo XXI". Desde que el Informe Delors (1996) cerró el siglo anterior indicando los pilares de la educación: aprender a conocer, a hacer, a ser y a vivir juntos, muchos otros han analizado las competencias personales, sociales, para el aprendizaje y para el trabajo que deberían orientar la educación de este nuevo siglo. Existen numerosos listados con este tipo de competencias. El más extendido es el que habla de las "cuatro ces": pensamiento crítico, creatividad, comunicación y colaboración. Pero en mi opinión estas cuatro competencias se quedan cortas, yo las completaría con otras dos absolutamente imprescindibles desde una formación más humanista que son: el conocimiento emocional y el compromiso.
El desarrollo del pensamiento crítico tiene que llevar a que nuestros alumnos reflexionen de forma activa, sean capaces de localizar información, seleccionarla, organizarla, analizarla, interpretar datos empíricos, discernir entre lo que es opinión, manipulación y los datos objetivos y utilizar esa información para tomar decisiones o resolver problemas. El pensamiento crítico va de la mano de la capacidad investigadora de los sujetos y esta capacidad debe utilizar los recursos tecnológicos del mundo de la información y la comunicación que tanto se han desarrollado en los últimos años. La capacidad investigadora va a terminar siendo uno de los ejes vertebrales del aprendizaje donde las habilidades informacionales y digitales del alumno serán claves.

La propuesta de soluciones creativas, originales e innovadoras a los problemas y a los retos debe potenciarse de forma planificada dentro de la formación. Los profesores deberían establecer las condiciones necesarias para que durante el aprendizaje los alumnos puedan hacer propuestas creativas ante nuevos retos y problemas. Es preciso fomentar el pensamiento divergente y apoyar al alumno hacia la innovación y el análisis de viabilidad de soluciones originales no previstas.

Aprender a pensar críticamente no necesariamente tiene que conseguirse de forma individual, todo lo contrario, el trabajo colaborativo, donde la diversidad de los grupos debería buscarse para que las diferentes miradas puedan estar presentes, llevaría a soluciones construidas a través de las aportaciones complementarias de los diferentes miembros de los equipos. Y todavía sería mejor si pudieran traspasarse las paredes del aula y trabajar en equipos de alumnos que pertenezcan a centros distintos, de países diferentes y culturas diversas. La riqueza de los aprendizajes que podrían tener los alumnos en esta red internacional e intercultural sería enorme.

Trabajar con otros, comunicarse y relacionarse con ellos les permitirá comprender mejor las diferencias. El conocimiento lleva al respeto y a la apertura de 
mente, lleva a poner la mirada más allá de uno mismo y permite ponerse en el lugar del otro. El respeto y conocimiento mutuo, la comprensión ante las diferencias fomentan el compromiso personal con la sociedad y con los problemas del entorno. El desarrollo de este compromiso permitirá a los alumnos utilizar sus conocimientos, inteligencia y creatividad a favor del bien común y de la mejora de nuestro mundo.

No podemos olvidarnos de la importancia del conocimiento emocional que Ileva implícito el trabajo considerando los sentimientos y las emociones propias y de los demás. El desarrollo de un adecuado conocimiento emocional lleva consigo la mejora en todas las habilidades sociales, permite una adecuada socialización y potencia las relaciones de convivencia y colaboración pacífica. Si el profesorado es consciente de sus propias emociones y trabaja con sus alumnos en este ámbito, los aprendizajes que se producirán serán realmente significativos. Los nuevos avances de la neurociencia y su aplicación a la educación lo confirman. El verdadero aprendizaje ocurre cuando al alumno se le presenta una nueva situación o contenido que es estimulante para él, que le provoca una reacción emocional, focalizando su atención e implicándole personalmente.

\section{Los maestros que liderarán el cambio}

Para que esta revolución sea realmente positiva necesitamos manos y cabezas que lideren el cambio, que lo hagan desde el conocimiento profundo de la realidad educativa de los centros y obviamente solo pueden hacer este papel los propios maestros. Estos maestros que serán el motor del cambio en la educación han aprendido como alumnos en un sistema educativo totalmente distinto al que van a tener que incorporarse como profesores. En este nuevo entorno, los alumnos aprenderán de forma distinta a como ellos lo hicieron, con recursos diferentes a los que ellos utilizaron y con objetivos y metodologías también diferentes. Necesitamos nuevos profesores para esta nueva edu-

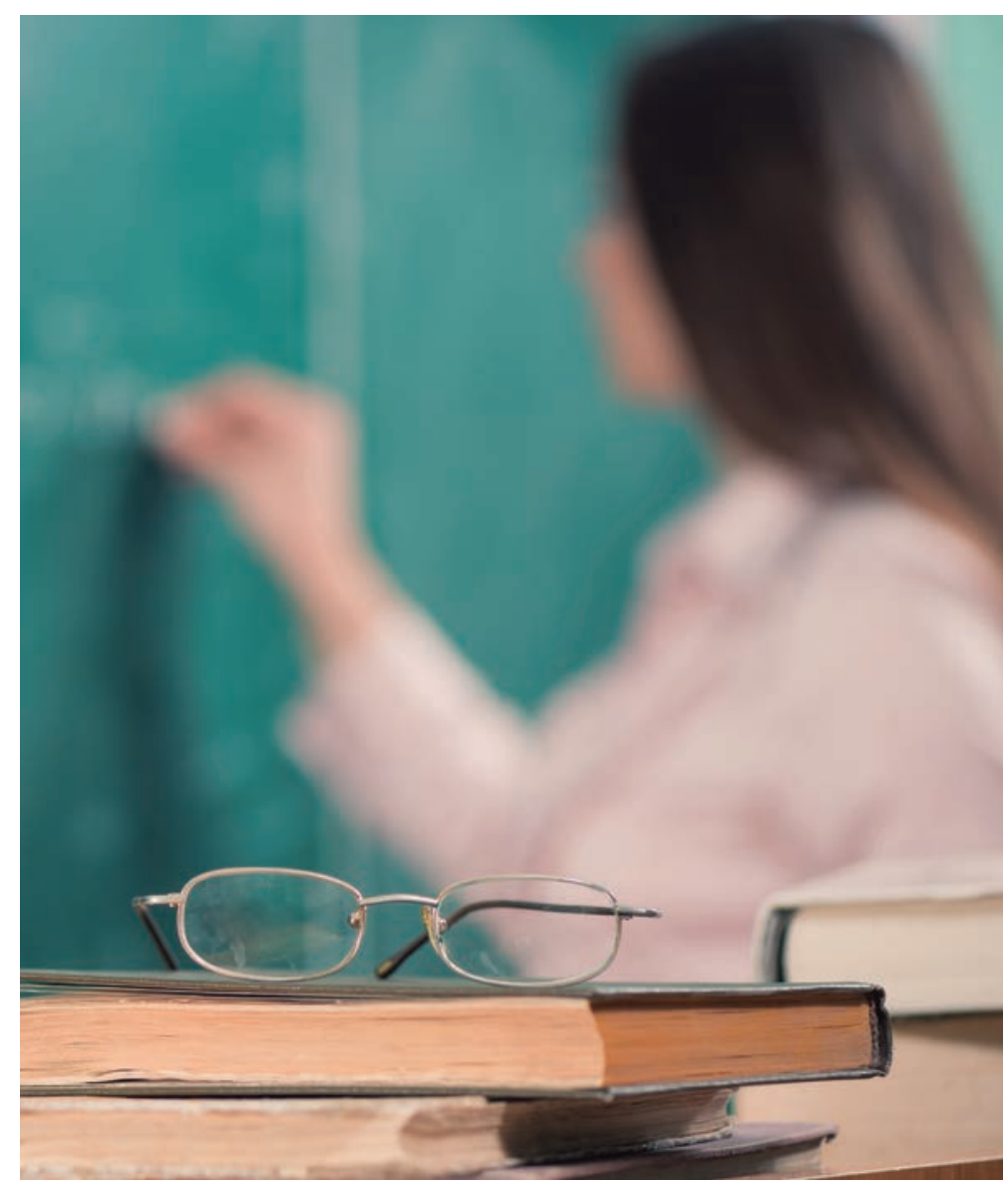

cación y no podemos seguir formándoles para el ejercicio profesional que deberán llevar a cabo en este siglo con maneras y enfoques del siglo XX. La generación de maestros que están actualmente en formación serán los que llevarán a cabo la revolución educativa, tendrán un duro papel de transición entre la antigua y la nueva educación.

Por tanto, es imprescindible que también reflexionemos sobre la formación de los maestros y revisemos el modelo. Si es urgente el cambio en los centros educativos, lo es más en la universidad. Es necesaria también una revolución en esta formación. No podemos replicar los errores del sistema educativo en los planes de estudios de la formación inicial de maestros. El análisis de esta formación debe llevarnos a reorganizar los contenidos, su estructura, pero sobre todo, la metodología didáctica que se emplea en los centros universitarios. Si queremos huir de la fragmentación disciplinar y fomentar el trabajo interdisciplinar, nuestros planes de estudio deben reorganizarse y rediseñar sus asignaturas, si queremos que los futuros maestros 


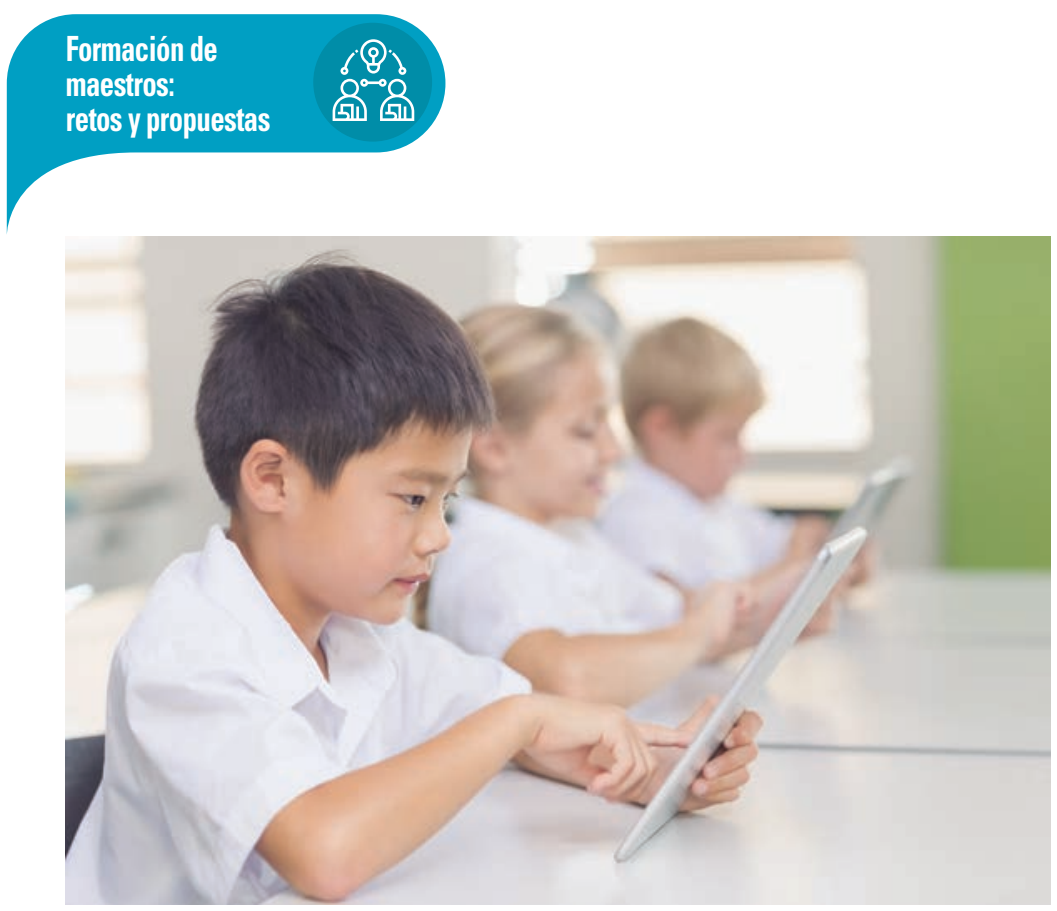

sepan fomentar a través de su actividad docente el pensamiento crítico, la creatividad, la colaboración, la comunicación, el conocimiento emocional y el compromiso, debemos dotarles de los recursos que les permitan elaborar propuestas didácticas adecuadas para ello. Y más aún formales a ellos mismos en el desarrollo de las competencias del siglo XXI.

Si la figura del maestro es la clave del éxito de este nuevo periodo debe potenciarse el que pueda tener un desarrollo profesional significativo. Si la sociedad es consciente de la necesidad de la revoIución educativa y del papel del maestro, es preciso que también lo reconozca y lo prestigie.

\section{Los maestros del siglo XXI}

Ante el nuevo contexto educativo que plantea este siglo deben redefinirse los roles y funciones del profesor y adecuar los planes de estudio de las titulaciones de Magisterio a este nuevo papel. Es preciso revisar qué debe saber y qué debe saber hacer este nuevo maestro y establecer el procedimiento más adecuado para que pueda conseguirlo.

Los maestros que cambiarán la educación deberían tener conocimientos teóricos y prácticos:

a) de las diferentes disciplinas y también integrados e interdisciplinares, que permita abordar los contenidos no de forma fragmentada sino atendiendo a los problemas y retos del futuro y del mundo;

b) del diseño didáctico necesario para la adquisición de las competencias de este siglo, y de las metodologías activas y participativas que potencien la adquisición de las competencias de nuestro siglo y concretamente de la metodología del aprendizaje basado en problemas, proyectos o retos que llevarán al alumno a enfrentarse a la aplicación real del conocimiento a la solución de problemas de la vida;

c) sobre el diseño y creación de contenidos y recursos para el aprendizaje, en diferentes soportes utilizando los recursos tecnológicos que propician los avances en esta área;

d) sobre neuroeducación, para entender la manera en la que aprende el cerebro y la aplicabilidad didáctica de sus avances teniendo en cuenta las diferentes etapas evolutivas del niño;

e) de las dificultades de aprendizaje y de la diversidad derivada de necesidades educativas específicas, que permita orientar de manera personalizada el aprendizaje al mismo tiempo que se trabaja de forma global con los alumnos:

f) emocionales, que los ayude a la gestión del aula y al apoyo al desarrollo individual de los alumnos y potencie sus habilidades inter e intrapersonales, que les permita entender la diversidad y enfocar sus diseños didácticos de forma inclusiva y los propicie a motivar de forma adecuada al alumnado;

g) de técnicas de liderazgo y conducción de grupos, que fomenten la colaboración y cooperación entre los alumnos y con otros profesores;

h) del método científico, que les permita llevar a cabo investigaciones sobre la eficacia de sus intervenciones y desarrollar su propio pensamiento crítico utilizando en la base datos empíricos y propicie a su vez el desarrollo de las destrezas de investigación en los alumnos;

i) transversales en idiomas, tecnológicos y de manejo de la información que les permita comunicarse de forma eficaz en contextos internacionales e interculturales, con una presencia activa en redes sociales, que les posi- 
bilite la participación activa en comunidades globales interconectadas de aprendizaje...

Pero deberían poder llevar a cabo su labor con iniciativa propia, innovación, pensamiento emprendedor, creatividad, desarrollando sus propias competencias metacognitivas, con autonomía, responsabilidad y compromiso, pero sobre todo con pasión hacia su labor educadora. El desarrollo como personas y como profesionales debería fomentarse desde la formación en su aprendizaje inicial.

\section{El cambio necesario en la formación de maestros}

Las universidades que formamos maestros estamos atados por las normativas que constriñen los planes de estudios $y$, a veces, estas imposibilitan el pensamiento creativo que propiciaría entornos más apropiados para el desarrollo de los aprendizajes que los profesores en formación necesitan para abordar el cambio educativo. Deberíamos utilizar un pensamiento abierto y flexible que nos permita manejar los espacios de libertad que proporcionan las normativas y, sobre todo, aproximar nuestro contexto educativo al que van a encontrarse en las escuelas. No se trata de escolarizar la universidad, sino de mantener el nivel académico utilizando metodologías que también propicien la adquisición de las competencias del siglo XXI en los profesores en formación para que a su vez sepan cómo fomentar en un futuro estas competencias entre sus propios alumnos.

Los grados de Magisterio deberían poderse llevar a cabo en entornos flexibles, donde la estructuración de horarios pudiera adaptarse a actividades interdisciplinares y colaborativas entre el profesorado, donde se pudieran abordar problemas reales de forma creativa utilizando los conocimientos de diferentes áreas, donde se puedan crear contenidos y materiales para el aprendizaje que no necesariamente pertenezcan a un único ámbito disciplinar. La propuesta de retos, proyectos o problemas debería ser la me-

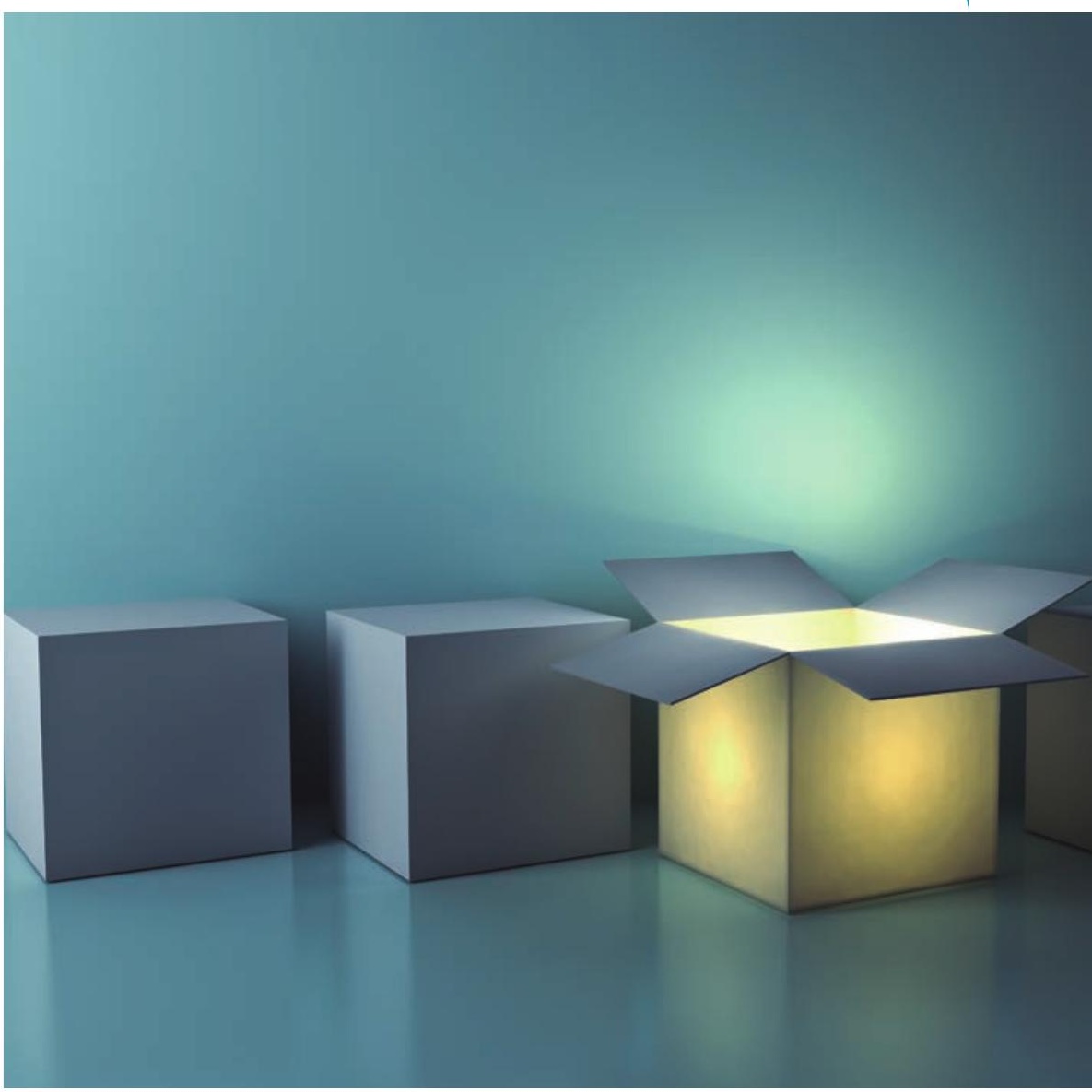

todología más común en este contexto. Debemos repensar las asignaturas, pero sobre todo las metodologías didácticas que empleamos.

La formación en el diseño didáctico debería ser uno de los ejes vertebrales de los estudios dado que es una de las competencias fundamentales del futuro profesor. No deberíamos pensar la formación didáctica de manera diferenciada, por disciplinas, sino de forma integrada, focalizándola hacia el ejercicio profesional futuro. Las prácticas profesionales en centros educativos de nuestros alumnos deberían ser el otro eje de la formación, pero unas prácticas que pudieran poner en funcionamiento diseños didácticos que surgieran desde el resto de las asignaturas, que permitieran innovar y emprender nuevas experiencias en los centros educativos y que Ilevaran a los alumnos de forma supervisada a la reflexión sobre los efectos de las diferentes intervenciones. Prácticas que pudieran simular el futuro, porque si los maestros en formación no pueden poner en práctica las intervenciones necesarias para la formación de los alumnos del siglo XXI, Io que realmente aprenderán será lo que los 
Los dos ejes vertebrales de los estudios de Magisterio deberían ser la formación para el diseño didáctico y las práclicas profesionales que lleven a la reflexión supervisada sobre los efectos que tienen en los alumnos las intervenciones educalivas

alumnos del siglo $X X$ necesitaron y esto no ayudará a que realmente se produzca el cambio necesario en la educación. Solo podremos llevar a cabo esta formación colaborando de forma estrecha y eficaz entre los centros educativos y la universidad, pensando juntos en el contexto adecuado para el desarrollo profesional de los maestros de este siglo.

El resto de las áreas de la formación de los maestros deberían aportar sus conocimientos a estos dos ejes principales: la formación didáctica y la formación práctica reflexionada. El nivel académico y formativo que se adquirirá de esta coordinación e integración de conocimientos tendrá como resultado una formación de alta calidad académica y profesional en los maestros.

Solo así conseguiremos que nuestros futuros maestros sean la clave de la revolución educativa porque solo así conseguiremos que tengan pensamiento crítico,

\section{DARA SABER MÂS}

PINEIDA, F. O. (2011). Competencies for the $21^{\text {st }}$ century: integrating ICT to life, school and economical development. Procedia. Social and Behavioral Sciences, 28, 54-57.

ScotT, C. L. (2015). ¿Por qué deben cambiar el contenido y los métodos de aprendizaje en el siglo XXI? París: UNESCO

ScotT, C. L. (2015). ¿Qué tipo de aprendizaje se necesita en el siglo XXI? París: UNESCO sean creativos, sepan comunicarse de forma adecuada, colaboren en comunidades globales interconectadas de aprendizaje, utilicen su inteligencia y conocimiento emocional y puedan comprometerse con ellos mismos y con un ejercicio profesional responsable. Y solo así podrán ellos provocar estos aprendizajes en sus alumnos y educar a los ciudadanos del futuro que necesita nuestra sociedad.

\section{Conclusiones}

En el nuevo contexto mundial fruto de la última revolución tecnológica, es necesario revisar los modelos educativos de formación que actualmente están presentes en la mayoría de los centros educativos. Es necesario que exista también otra revolución, la revolución en educación. Debemos introducir las modificaciones necesarias en el currículo y sobre todo en la metodología didáctica que propicie el que los alumnos lleguen a alcanzar las competencias que les serán demandadas en este siglo como ciudadanos activos y proactivos ante los problemas acuciantes de nuestro mundo. Lo que más urge en este momento es formar adecuadamente a los maestros que liderarán el cambio educativo y para ello es necesario transformar la formación inicial universitaria que reciben. Las universidades tenemos la responsabilidad de adaptarnos a este contexto innovador que, aunque tenga un alto grado de incertidumbre, está lleno de oportunidades que debemos saber aprovechar •

HEMOS HABLADO DE

Formación de maestros; metodologías de enseñanza; competencias; aprendizaje basado en problemas; estrategias educativas.

Este artículo fue solicitado por PADRES Y MAESTROS en septiembre de 2019, revisado y aceptado en febrero de 2020. 